\title{
The effect of pelvic floor muscle exercises on sexual function in women with multiple sclerosis: a pre-/post-intervention clinical trial
} \author{
NOSRAT ZAHERIAN ${ }^{1, A-F}$, PARVANEH MOUSAVI 2, B-F, NASTARAN MAJDINASAB ${ }^{3, \text { c-F }}$, \\ MOHAMMAD HOSSEIN HAGHIGHIZADEH ${ }^{4, C-E}$, POORANDOKHT AFSHARI, D-F
}

\author{
${ }^{1}$ Reproductive Health Promotion Research Center, School of Nursing and Midwifery, Ahvaz Jundishapur University \\ of Medical Sciences, Ahvaz, Iran \\ ${ }^{2}$ Faculty of Nursing and Midwifery, Menopause Research Center, Ahvaz Jundishapur University of Medical Sciences, \\ Ahvaz, Iran \\ ${ }^{3}$ Comprehensive Rehabilitation Research Center, Ahvaz Jundishapur University of Medical Sciences, Ahvaz, Iran \\ ${ }^{4}$ Department of Statistics and Epidemiology, School of Public Health, Ahvaz Jundishapur University of Medical \\ Sciences, Ahvaz, Iran
}

A - Study Design, B - Data Collection, C - Statistical Analysis, D - Data Interpretation, E - Manuscript Preparation, F - Literature Search, G - Funds Collection

Summary Background. Sexual dysfunction (SD) is a common symptom in patients with multiple sclerosis (MS). In women with MS there are no specific pharmacological or non-pharmacological therapies which have been proven effective for SD.

Objectives. The main objective of this study was to evaluate the effect of pelvic floor muscle exercises on improving sexual function in women with MS.

Material and methods. This study was a pre-/post-intervention clinical trial conducted on $45 \mathrm{MS}$ women who had been referred to the MS Clinic in Ahvaz, Iran. All participants were selected by the purposeful sampling method. The data collection tools in this study included a demographic data form and the Female Sexual Function Index (FSFI) questionnaire. The study participants $(n=45)$ performed pelvic floor muscle exercises for 12 consecutive weeks. The data were analyzed as descriptive statistics (mean and standard deviation) with the Shapiro-Wilk test, the independent $t$-test, and the paired $t$-test using SPSS software version 24 ; a $p$-value of $<0.05$ was selected as the significance level.

Results. The results revealed that after pelvic floor muscle exercise, the mean score of sexual function of women with MS had increased from 10.58 to 30.17 and that this increase was statistically significant $(p=0.001)$.

Conclusions. Pelvic floor muscle exercise can improve sexual function in women with MS. Thus, performing these exercises is recommended as an easy and cost-effective method to improve the sexual function of these patients.

Key words: sexuality, pelvic floor, multiple sclerosis, women.

Zaherian N, Mousavi P, Majdinasab N, Haghighizadeh MH, Afshari P. The effect of pelvic floor muscle exercises on sexual function in women with multiple sclerosis: a pre-/post-intervention clinical trial. Fam Med Prim Care Rev 2020; 22(2): 179-182, doi: https://doi. org/10.5114/fmpcr.2020.95328.

\section{Background}

Multiple sclerosis (MS) is the most common chronic nervous system disorder leading to disability and dysfunction in young people $[1,2]$. It is most commonly seen in people between the ages of 20 and 45 years [1, 3]. The incidence of MS in women is three times higher than that in men $[4,5]$. Reports have suggested that about 2.5 million people suffer from MS around the world [1].

This autoimmune disease is characterized by a variety of unpredictable signs and symptoms, such as muscle cramps, fatigue, pain, visual disturbances, dysphagia, speech and breathing disorders, bladder and intestinal problems, cognitive disorders, and sexual problems [6-9].

Sexual dysfunction (SD) is an important public health concern and a common symptom in MS patients, with a prevalence of $40 \%$ to $80 \%[9,10]$. Previous studies have suggested the incidence rate of SD to be $31 \%$ among Iranian females, but the prevalence of SD in Iranian women with MS is unknown [11].
Since MS occurs in ages at which patients are sexually active, it is important to find appropriate therapies to enhance their sexual function.

Pelvic floor muscles (PFMs) are known to play a key role in sexual function, as they are responsible for the involuntary rhythmic contractions during orgasm and for vaginal sensation during intercourse $[12,13]$.

One of the methods for strengthening the PFMs is a pelvic floor exercise that is also known as Kegel exercises [14, 15]. Kegel exercises involve repeated voluntary and selective contraction and relaxation of specific muscles in the pelvic floor [1618]. It is argued that the use of PFM exercise might contribute to the involuntary contractions in women during intercourse and may increase the blood flow to the pelvic floor muscles, resulting in better sexual activity in couples [19]. In women with MS, there are no specific pharmacological or non-pharmacological therapies which have been proven effective in sexual dysfunction. Previous studies have shown the effects of PFM training in the treatment of SD in women suffering from MS [20]. 


\section{Objectives}

The aim of this study is to assess the efficiency of Kegel exercises as a safe and effective method for improving the sexual function in women suffering from multiple sclerosis.

\section{Material and methods}

\section{Ethical consideration}

This study was approved by the Ethics Committee of Ahvaz Jundishapur University of Medical Sciences, Ahvaz, Iran in accordance with the Declaration of Helsinki (Ref No: IR.AJUMS. REC.1397.312). After explaining the aims of this research to all participants, their written informed consent was obtained.

\section{Study design and data collection}

This study was a pre-/post-intervention clinical trial conducted on MS women referred to the MS Clinic in Ahvaz, in southwestern Iran. The data were collected through a demographic data form and the Female Sexual Function Index (FSFI) questionnaire. The demographic data form included age, spouse's age, marriage duration, duration of disease, number of pregnancies, number of childbirths, type of delivery, educational level, job, spouse's educational level, spouse's job, economic situation, type of housing, type of disease, and the number of disease recurrences. The FSFI questionnaire was developed and validated in 2000 [21]. It contains 19 questions, including 2 questions concerning libido, 4 questions about sexual arousal, 4 questions regarding lubrication, and 3 questions each for orgasm, sexual satisfaction, and pain. A five-point Likert scale was used for scoring. The score of each domain was multiplied by a certain factor. The factor for desire was 0.6 , for arousal and moisture was 0.3 , and for other domains was 0.4 . A total sum of each score is calculated, and higher scores indicate healthier sexual function. The scores range between 1.2 and 36 points [22]. In Iran, Mohammadi et al. calculated the reliability of the scale and subscales to be 0.85 through Cronbach's alpha coefficient for all individuals. In this study, the appropriate cut-off score of the whole scale for the diagnosis of sexual dysfunction was determined to be 28 points [23].

\section{Participants and sampling method}

In this study, 52 women with MS were selected by purposeful sampling. The sample size was determined using a $95 \%$ confidence interval, $80 \%$ power, and considering the probability of a $10 \%$ dropout rate in the PFM exercise group, according to the protocol of Machin et al. [24]. Accordingly, the sample size was determined using G Power package software version 3.1.

\section{Inclusion and exclusion criteria}

The inclusion criteria included an age range of $18-45$ years, reading and writing literacy, being married, a diagnosis of multiple sclerosis, and a sexual function score below 26.5 on the FSFI questionnaire as administered by a neurologist. The exclusion criteria included severe cognitive problems, a disease recurrence in the previous three months, excessive fatigue preventing the person from performing the exercises, pelvic organ prolapse, and previous gynecological surgeries.

\section{Intervention protocol}

The study participants performed PFM exercises for 12 consecutive weeks. Kegel exercises were recommended to patients three times a day: morning, evening, and night. For each session, the subjects contracted their pelvic floor muscles five times. They were also recommended to contract and relax their pelvic floor muscles 10 to 20 times immediately and without interruption after sexual intercourse, after completing urination, and between their daily activities. The PFM exercise group was followed up regularly by the researcher. Finally, the researcher re-administered the demographic and FSFI questionnaires after 12 weeks of the PFM exercise program. Any subject who had not regularly, daily exercised at home, or who experienced muscle weakness, blurred vision, or a lack of coordination, was excluded from the study.

\section{Analysis of data}

All statistical analyses were done using SPSS ${ }^{\mathrm{TM}}$ software version 24.0 (IBM Corporation, Armonk, NY, USA). An independent $t$-test was used to compare sexual function scores. A $p$-value of $<0.05$ was considered to be the level of significance.

\section{Results}

In this study, out of 52 participants a total of 45 women with MS met the inclusion criteria. The mean age of the subjects was $35.71 \pm 6.61$ years. Tables 1 and 2 present the quantitative and qualitative demographic information of the study participants, respectively. Based on the results listed in Table 3, the total score of sexual function in the women with MS was 10.58 points before the intervention, which increased to 30.17 points after the intervention; this increase was statistically significant $(p=0.001)$. With regard to each dimension of sexual function, the results were as follows: the desire score increased from 2.04 to 4.72 points, the arousal score increased from 1.81 to 4.80 points, the vaginal moisture score increased from 1.73 to 5.08 points, the orgasm score increased from 1.60 to 5.16 points, the sexual satisfaction score increased from 1.93 to 5.92 points, and the pain score increased from 1.81 to 5.03 points after the intervention $(p<0.05)$.

Table 1. Comparison of the mean and standard deviation of the quantitative variables of the participants

\begin{tabular}{|l|l|}
\hline Variable & Mean \pm SD \\
\hline Age (years) & $35.71 \pm 6.61$ \\
\hline Spouse's age (years) & $42.69 \pm 8.86$ \\
\hline Marriage duration (years) & $14.07 \pm 8.82$ \\
\hline Number of pregnancies & $2.18 \pm 1.64$ \\
\hline Number of childbirths & $1.89 \pm 1.31$ \\
\hline Disease duration (years) & $6.04 \pm 3.33$ \\
\hline
\end{tabular}

Table 2. Frequency distribution and percentage of qualitative variables related to the participants

\section{Variable} $n(\%)$

\begin{tabular}{|l|l|l|}
\hline \multirow{5}{*}{ Type of delivery } & caesarean & $17(37.8)$ \\
\cline { 2 - 3 } & natural with episiotomy & $11(24.4)$ \\
\cline { 2 - 3 } & natural childbirth & $13(28.9)$ \\
\cline { 2 - 3 } & no childbirth & $4(8.9)$ \\
\hline \multirow{5}{*}{ Pducational level } & elementary school & $14(31.1)$ \\
\cline { 2 - 3 } & secondary school & $6(13.3)$ \\
\cline { 2 - 3 } & high school & $8(17.8)$ \\
\cline { 2 - 3 } & academic & $17(37.8)$ \\
\hline \multirow{2}{*}{$\begin{array}{l}\text { Educational level of } \\
\text { spouse }\end{array}$} & employee & $15(33.3)$ \\
\cline { 2 - 3 } & housewife & $20(66.7)$ \\
\cline { 2 - 3 } & elementary school & $2(4.4)$ \\
\cline { 2 - 3 } & secondary school & $9(20)$ \\
\cline { 2 - 3 } & high school & $13(28.9)$ \\
\cline { 2 - 3 } & academic & $21(46.7)$ \\
\hline
\end{tabular}




\begin{tabular}{|c|c|c|}
\hline \multicolumn{2}{|l|}{ Variable } & \multirow{2}{*}{\begin{tabular}{|l|}
$n(\%)$ \\
$19(42.2)$ \\
\end{tabular}} \\
\hline \multirow[t]{3}{*}{ Spouse's job } & employee & \\
\hline & self-employed & $20(44.4)$ \\
\hline & unemployed & $6(13.3)$ \\
\hline \multirow[t]{3}{*}{ Economic level } & low & $11(24.4)$ \\
\hline & average & $28(62.2)$ \\
\hline & high & $6(13.3)$ \\
\hline \multirow[t]{2}{*}{ Type of housing } & owner & $27(62.2)$ \\
\hline & tenant & $17(37.8)$ \\
\hline \multirow[t]{2}{*}{ Type of disease } & relapsing-remitting & $29(64.4)$ \\
\hline & secondary-progressive & $16(35.6)$ \\
\hline \multirow{4}{*}{$\begin{array}{l}\text { Number of disease } \\
\text { recurrences }\end{array}$} & recurring & $24(53.3)$ \\
\hline & one & $15(33.3)$ \\
\hline & two & $4(8.9)$ \\
\hline & more than two & $2(4.6)$ \\
\hline
\end{tabular}

\begin{tabular}{|c|c|c|c|}
\hline Variable & $\begin{array}{l}\text { Before inter- } \\
\text { vention } \\
\text { (Mean } \pm \text { SD) } \\
\end{array}$ & $\begin{array}{l}\text { After interven- } \\
\text { tion } \\
\text { (Mean } \pm \text { SD) } \\
\end{array}$ & $p$ \\
\hline Sexual desire & $2.04 \pm 0.78$ & $4.72 \pm 0.87$ & 0.001 \\
\hline Sexual arousal & $1.81 \pm 0.87$ & $4.8 \pm 0.8$ & 0.001 \\
\hline Vaginal moisture & $1.73 \pm 0.79$ & $5.08 \pm 0.65$ & 0.001 \\
\hline Orgasm & $1.6 \pm 0.72$ & $5.16 \pm 0.54$ & 0.001 \\
\hline $\begin{array}{l}\text { Sexual satisfac- } \\
\text { tion }\end{array}$ & $1.93 \pm 0.58$ & $5.42 \pm 0.54$ & 0.001 \\
\hline Sexual pain & $1.81 \pm 0.88$ & $5.03 \pm 0.74$ & 0.001 \\
\hline \begin{tabular}{|l|}
$\begin{array}{l}\text { Total sexual func- } \\
\text { tion }\end{array}$ \\
\end{tabular} & $10.58 \pm 4.01$ & $30.17 \pm 3.4$ & 0.001 \\
\hline
\end{tabular}

\section{Discussion}

The results reveal that sexual function in women with MS was significantly improved after PFM exercise intervention. The sexual function score was 10.58 before the intervention, and it increased to 30.17 afterwards. The results of this study are consistent with the findings of Zohariou et al., which showed that PFM exercise improved the total score of sexual function from $20.3 \pm 2.3$ to $26.8 \pm 2.8$ in women with stress urinary incontinence after 12 months of intervention [16]. These effects seem to be due to the strengthening of the muscles attached to the clitoris, which can improve pelvic floor muscle involuntary con- traction during orgasm by improving pelvic blood flow, pelvic mobility, and clitoral sensitivity. In fact, increased blood flow to the pelvis and increased clitoral sensitivity can improve sexual arousal, vaginal moisture, and orgasm [19].

Several reports from different countries have shown the efficacy of PFM exercises in improving sexual function in female patients, but so far few studies have examined the effect of these exercises on the sexual function of MS patients. In a study conducted by Citak et al., the results showed that PFM exercises did not increase sexual satisfaction, but they did have a positive effect on pelvic muscle function and female sexual function [25]. Thus, their results were consistent with those of the current study. In another study conducted by Pourkhiz et al., PFM exercises were able to improve female sexual function during pregnancy and delivery, findings that are in line with the current study [19]. In a study conducted by Zare et al., who evaluated the effect of PFM exercise on quality of life and marital satisfaction in primiparous women after delivery, the quality of sexual life in both the intervention and control groups had significantly improved eight weeks after the beginning of the study [26]. However, in contrast with the aforementioned studies, Lara et al. reported that PFM exercises were effective in strengthening these muscles, but they did not affect sexual function [27]. This discrepancy might be due to differences in the studied population, the sample size, sampling method, and measurement tools.

In one of the few studies conducted on MS patients, Lúcio et al. reported that PFM exercises alone or combination with electrostimulation have positive effects on the sexual function of women suffering from MS [20]. In their study, all evaluated groups showed an improvement overall and in the arousal, lubrication, and satisfaction domains of the FSFI questionnaire - this is in line with the current study.

\section{Limitations of the study}

In this study, the study group did not reach the specified number, due to some of the MS patients not qualifying for participation in the study.

\section{Conclusions}

PFM exercise is an effective method to increase sexual function in women with MS, and given its low cost and non-invasive nature, it is recommended to use Kegel exercises as a useful and effective method to improve sexual function in MS patients in general practice.

Acknowledgments. This paper was part of the master's thesis of Nosrat Zaherian, which was approved and financially supported by a grant (No: PHPRC-9704) from the Research affairs of Ahvaz Jundishapur University of Medical Sciences, Ahvaz, Iran.

Source of funding: This work was funded from Research affairs of Ahvaz Jundishapur University of Medical Sciences, Ahvaz, Iran. Conflicts of interest: The authors declare no conflicts of interest.

\section{References}

1. Huang WJ, Chen WW, Zhang X. Multiple sclerosis: pathology, diagnosis and treatments. Exp Ther Med 2017; 13(6): 3163-3166.

2. Filippi M, Bar-Or A, Piehl F, et al. Multiple sclerosis. Nat Rev Dis Primers 2018; 4(1): 49.

3. Dobson R, Giovannoni G. Multiple sclerosis - a review. Eur J Neurol 2019; 26(1): 27-40.

4. Houtchens MK, Edwards NC, Schneider G, et al. Pregnancy rates and outcomes in women with and without MS in the United States. Neurology 2018; 91(17): e1559-e1569.

5. Azimian M, Shahvarughi-Farahani A, Rahgozar M, et al. Fatigue, depression, and physical impairment in multiple sclerosis. Iran J Neurol 2014; 13(2): 105-107.

6. Christogianni A, Bibb R, Davis SL, et al. Temperature sensitivity in multiple sclerosis: an overview of its impact on sensory and cognitive symptoms. Temperature 2018; 5(3): 208-223.

7. Goldenberg MM. Multiple sclerosis review. P T 2012; 37(3): 175-184. 
8. Gross HJ, Watson C. Characteristics, burden of illness, and physical functioning of patients with relapsing-remitting and secondary progressive multiple sclerosis: a cross-sectional US survey. Neuropsychiatr Dis Treat 2017; 13: 1349-1357.

9. Pöttgen J, Rose A, van de Vis W, et al. Sexual dysfunctions in MS in relation to neuropsychiatric aspects and its psychological treatment: a scoping review. PLOS ONE 2018; 13(2): e0193381.

10. Flynn KE, Lin L, Bruner DW, et al. Sexual satisfaction and the importance of sexual health to quality of life throughout the life course of U.S. adults. J Sex Med 2016; 13(11): 1642-1650.

11. Mohammadi K, Rahnama P, Mohseni SM, et al. Determinants of sexual dysfunction in women with multiple sclerosis. BMC Neurol 2013; 13: 83, doi: 10.1186/1471-2377-13-83.

12. Sobhgol SS, Priddis $\mathrm{H}$, Smith CA, et al. Evaluation of the effect of an antenatal pelvic floor muscle exercise programme on female sexual function during pregnancy and the first 3 months following birth: study protocol for a pragmatic randomised controlled trial. Trials 2019; 20(1): 144, doi 10.1186/s13063-019-3226-6.

13. Ozdemir FC, Pehlivan E, Melekoglu R. Pelvic floor muscle strength of women consulting at the gynecology outpatient clinics and its correlation with sexual dysfunction: a cross-sectional study. Pak J Med Sci 2017; 33(4): 854-859.

14. Ptak M, Ciećwież S, Brodowska A, et al. The effect of pelvic floor muscles exercise on quality of life in women with stress urinary incontinence and its relationship with vaginal deliveries: a randomized trial. BioMed Res Int 2019; 2019: 5321864, doi: 10.1155/2019/5321864.

15. Golmakani N, Zare Z, Khadem N, et al. The effect of pelvic floor muscle exercises program on sexual self-efficacy in primiparous women after delivery. Iran J Nurs Midwifery Res 2015; 20(3): 347-353.

16. Zahariou AG, Karamouti MV, Papaioannou PD. Pelvic floor muscle training improves sexual function of women with stress urinary incontinence. Int Urogynecol J Pelvic Floor Dysfunct 2008; 19(3): 401-406.

17. Braekken IH, Majida M, Ellstrom Engh M, et al. Can pelvic floor muscle training improve sexual function in women with pelvic organ prolapse? A randomized controlled trial. J Sex Med 2015; 12(2): 470-480.

18. Hoseinkhani M, Taghian F. Effects of Kegel, central, and combined stability exercises on the central muscle endurance and quality of life of primiparous women after episiotomy. Iranian Journal of Obstetrics, Gynecology and Infertility 2018; 21(2): 60-68.

19. Pourkhiz Z, Mohammad-Alizadeh-Charandabi S, Mirghafourvand M, et al. Effect of pelvic floor muscle training on female sexual function during pregnancy and postpartum: a randomized controlled trial. Iran Red Crescent Med J 2017; 19(10): e63218.

20. Lúcio AC, D'Ancona CA, Lopes $\mathrm{MH}$, et al. The effect of pelvic floor muscle training alone or in combination with electrostimulation in the treatment of sexual dysfunction in women with multiple sclerosis. Mult Scler J 2014; 20(13): 1761-1768.

21. Pangastuti N, Santoso BI, Agustiningsih D, et al. Validation test of Indonesian female sexual function index (Indonesian FSFI). Bali Med J 2019; 8(1): 164-168.

22. Kayataş S, Özkaya E, Api M, et al. Comparison of libido, Female Sexual Function Index, and Arizona scores in women who underwent laparoscopic or conventional abdominal hysterectomy. Turk J Obstet Gynecol 2017; 14(2): 128-132.

23. Mohammadi K, Heydari M, Faghihzadeh S. The female sexual function index (FSFI): validation of the Iranian version. Payesh 2008; 7 : 269-278.

24. Machin D, Campbell M, Tan BT, et al. Sample size tables for clinical studies. 3rd ed. Wiley-Blackwell; 2009.

25. Citak N, Cam C, Arslan H, et al. Postpartum sexual function of women and the effects of early pelvic floor muscle exercises. Acta Obstet Gynecol Scand 2010; 89(6): 817-822.

26. Zare Z, Golmakani N, Khadem N, et al. The effect of pelvic floor muscle exercises on sexual quality of life and marital satisfaction in primiparous women after childbirth. Iran J Obstet Gynecol Infert 2014; 17(103): 21-32.

27. Lara LA, Montenegro ML, Franco MM, et al. Is the sexual satisfaction of postmenopausal women enhanced by physical exercise and pelvic floor muscle training? J Sex Med 2012; 9(1): 218-223.

Tables: 3

Figures: 0

References: 27

Received: 1.10 .2019

Reviewed: 24.10 .2019

Accepted: 6.02 .2020

Address for correspondence:

Pourandokht Afshari, MSc, Assoc. Prof.

Department of Midwifery, Reproductive Health Promotion Research Center

Ahvaz Jundishapur University of Medical Sciences

Golestan street

Postal Code: 61357-15794

P.O. Box: 159

Ahvaz, Khozestan

Iran

Tel.: +98 6133376689

E-mail: porandokhtafshari@gmail.com 